\title{
New data on the distribution of digger wasps of the genus Ammophila W. Kirby, 1798 (Hymenoptera, Sphecidae)
}

\author{
R.T-o. Baghirov ${ }^{1,2}$, A.A. Nesterovich ${ }^{1}$ \\ ${ }^{1}$ Tomsk State University, Lenin Ave. 36, Tomsk 634050 Russia. \\ 2 Siberian State Medical University, Moscow Road 2, Tomsk 634050 Russia. \\ E-mail:rbaghirov@yandex.ru
}

An annotated list is given and the distribution of five species of digger wasps genus Ammophila, collected in the circumpolar regions of Western Siberia (in the Yamalo-Nenets Autonomous District) in 2017 is specified.

Key words: Hymenoptera; Sphecidae; Ammophila; circumpolar regions; Western Siberia

\section{Introduction}

Sphecidae - mainly large, actively flying, clearly visible insects. Sphecidae make their nests in the ground, often in sandy soils. Most of Sphecidae prefer the open landscapes; they are rare in forests. The family is distributed worldwide, mainly in arid, semiarid and tropical regions. Number of taxa in Russian territory - 11 genus and 68 species. (Antropov et al., 2017).

The relevance of our work is dictated by the lack of literary information on the fauna of digger wasps of circumpolar regions. In the world scientific literature, so far there is no information on the fauna of Sphecidae of the circumpolar regions, for this reason we have planned and carried out this work.

\section{Material and methods}

The material was collected in the vicinity of the village of Khanymey (Purovsky District, Yamalo-Nenets Autonomous District) from 7.07 to 31.07.2017 in eight localities (Point No. 1-8). Baghirov collector unless otherwise specified. The abbreviations used in the text to designate places of assembly:

(Point №1) - 24.07.2017. Yamalo-Nenets Autonomous District, vicinity of the village of Khanymey, $60 \mathrm{~km}$ to the North, N 6353'33,2" E 7258'36,9" 79 m. above sea level. Material collected from the blossoming Epilobium angustifolium L., 1753;

(Point №2) - 26.07.2017. Yamalo-Nenets Autonomous District, vicinity of the village of Khanymey, N 6346'17,5" $E$ 76³2'10,2" $70 \mathrm{~m}$. above sea level. Material collected from the blossoming Epilobium angustifolia;

(Point №3) - 27.07.2017. Yamalo-Nenets Autonomous District, vicinity of the village of Khanymey, N 6343'15,6" E 7358'23,8" $69 \mathrm{~m}$. above sea level. Material collected from the blossoming Epilobium angustifolia;

(Point №4) - 28.07.2017. Yamalo-Nenets Autonomous District, vicinity of the village of Khanymey, N 6346'39,9" E 76³0'55,2" $67 \mathrm{~m}$. above sea level. Material collected from the blossoming Epilobium angustifolia;

(Point №5) - 28.07.2017. Yamalo-Nenets Autonomous District, vicinity of the village of Khanymey, N 6349'26,9" E $76^{\circ} 32^{\prime} 02,0^{\prime \prime} 60 \mathrm{~m}$. above sea level. Material collected from the blossoming Epilobium angustifolia;

(Point №6) - 29.07.2017. Yamalo-Nenets Autonomous District, vicinity of the village of Khanymey, $70 \mathrm{~km}$ road to Salekhard, N 6357'05,9" E 7454'32,9" 78 m. above sea level. Material collected from the blossoming Epilobium angustifolia;

(Point №7) - 29.07.2017. Yamalo-Nenets Autonomous District, vicinity of the village of Khanymey, road to Salekhard, N 6405'20,7" E 7501'55,1" 9595 m. above sea level. Material collected from a flowering field Leucanthemum vulgare Lam. (1779) and Trifolium pratense L., 1753;

(Point №8) - 31.07.2017. Yamalo-Nenets Autonomous District, village of Khanymey, N 6343'44,1" E 7556’53,7". Material collected from a flowering field Tanacetum vulgare L. 1753, Leucanthemum vulgare and Trifolium pratense;

The material was collected with the aid of mowing with an entomological net and using Merike cups. 
Baghirov, R.T-o., \& Nesterovich, A.A. New data on the distribution of genus Ammophila... Acta Biologica Sibirica, 2019, 5(1), 44-46

Systematics Sphecidae, as well as information about their distribution are given in accordance with the Catalog of Sphecidae (Pulawski, 2018). Species identification was carried out using a keys to genera and species (Danilov, 2014).

The abbreviations used in the text to refer to the distribution of species:

RUSSIA EUROPEAN PART (EP): North (N): Murmansk Prov., Arkhangelsk Prov., Karelian Rep., Vologda Prov., Komi Rep.; North-West (NW): Kaliningrad Prov., Leningrad Prov., Pskov Prov., Novgorod Prov.; Centre (C): Tver Prov., Yaroslavl Prov., Kostroma Prov., Smolensk Prov., Moscow Prov., Vladimir Prov., Ivanovo Prov., Nizhniy Novgorod Prov., Kaluga Prov., Tula Prov., Ryazan Prov., Mordovian Rep., Bryansk Prov., Orel Prov., Lipetsk Prov., Tambov Prov., Penza Prov., Kursk Prov., Belgorod Prov., Voronezh Prov.; East (E): Kirov Prov., Udmurt Rep., Mari El Rep., Chuvash Rep., Tatar Rep., Ulyanovsk Prov., SamaraProv., Saratov Prov.; South (S): Rostov Prov., Volgograd Prov., Kalmyk Rep., Astrakhan Prov.; North Caucasus (NC): Krasnodar Terr., Stavropol Terr., Adygei Rep., Karachayevo-Cherkess Rep., Ingush Rep., Kabardino-Balkarian Rep., North Ossetian Rep., Chechen Rep., Dagestan Rep.; Crimea (CR): Crimea Rep., Sebastopol.

URALS (UR): Perm Terr., Sverdlovsk Prov., Bashkir Rep., Chelyabinsk Prov., Orenburg Prov., Kurgan Prov.

WESTERN SIBERIA (WS): Tyumen Prov. (TM), Omsk Prov. (OM), Tomsk Prov. (TK), Novosibirsk Prov. (NS), Kemerovo Prov. (KM), Altai (AL) (including Altai Rep. and Altai Terr.).

EASTERN SIBERIA (ES): Khakass Rep. (KS), Tuva Rep. (TU), Krasnoyarsk Terr. (KR), Irkutsk Prov. (IR), Buryat Rep. (BR), Yakutsk Rep. (YA), Zabaikalskii Terr. (ZB);

FAR EAST (FE):Amur Prov. (AM), Khabarovsk Terr. (including Jewish Autonomous Region) (KH), Primorskii Terr. (PR),

Sakhalin (SA), Kuril Islands (KU), Kamchatka Terr. (KA), Magadan Prov. (MG), Chukot Autonomous Area (CH).

OTHER COUNTRIES: IR - Iran; KP - Korean Peninsula; KZ - Kazakhstan; MN - Mongolia; SY - Syria; TR - Turkey.

CHINA: Northeastern Territory (NE), Northern Territory (NC), Northwestern Territory (NW), the Western Plateau (WP), Central Territory (CC), Southwestern Territory (SW), Southeastern Territory (SE).

\section{Results}

We found 5 species of Ammophila in the study area. All species found by us are listed for the first time for the study area.

Sphecidae Latreille, 1802 (sensu stricto)

Ammophilinae André, 1886

Ammophilini

Ammophila W. Kirby, 1798

Miscus Jurine, 1807; Coloptera Latreille, 1845; Argyrammophila Gussakovskij, 1928; Apycnemia Leclercq, 1961. Type species Sphex sabulosus Linnaeus, 1758.

1. Ammophila asiatica Tsuneki, 1971

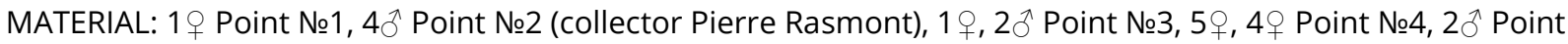

№5(collector Baptiste Martinet), 3ㅇ, 80 Point №6, 1 ㅇ, 1 त Point №7.

DISTRIBUTION: Russia: WS (AL), ES (TU, BR, ZB). - MN.

2. Ammophila campestris Latreille, 1809

Ammophilus retusus Gistel, 1848; Miscus neoxenus F. Smith, 1856; Ammophila slovaca Zavadil in Zavadil, Šustera, and Bat'a, 1937.

MATERIAL: 1 + Point №5.

DISTRIBUTION: Russia: EP (C, E, S, NC, CR), UR, WS (OM, TK, NS, AL), ES (TU, KR, IR), FE (AM, PR). - Europe (WE, NE, SE, EE), N Africa, TR, Central Asia, KZ, MN, China (NE, NC, NW), KP.

\section{Ammophila pubescens Curtis, 1836}

Miscus arvensis Dahlbom, 1843; Amophila susterai Šnoflák, 1943; Ammophila campestris var. alpicola de Beaumont, 1945; Ammophila adriaansei Wilcke, 1945; Ammophila arnaudi Tsuneki, 1976.

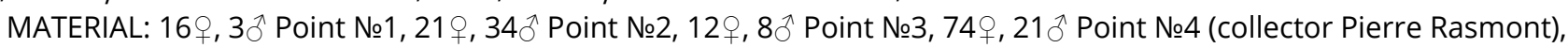

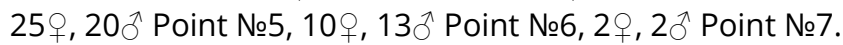

DISTRIBUTION: Russia: EP (N, NW, C, E, NC, CR), UR, WS, ES, FE (AM, KH, PR). - Europe (WE, NE, SE, EE), TR, IR, KZ, MN, China (NE, NC, NW, SW), N America.

\section{Ammophila sabulosa (Linnaeus, 1758)}

Sphex sabulosus Linnaeus, 1758; Sphex hortensis Poda von Neuhaus, 1761; Ichneumon frischii Geoffroy in Fourcroy, 1785; Sphex dimidiatus Christ, 1791; Ammophila vulgaris W. Kirby, 1798; Ammophila pu/villata Sowerby, 1806; Sphex mucronatus Jurine, 1807; Ammophila cyanescens Dahlbom, 1845; Ammophila vischu Cameron, 1889; Ammophila sabulosa kamtschatica Gussakovskij, 1932.

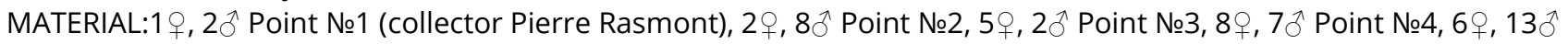
Point №5, 10우, 11 个 Point №6, 7우, 6ð Point №7.

DISTRIBUTION: Russia: EP, UR, WS, ES, FE (KA, MG). - Europe (WE, NE, SE, EE), N Africa, TR, SY, IR, Central Asia, KZ, MN, China (NC).

\section{Ammophila terminata F. Smith, 1852}


Baghirov, R.T-o., \& Nesterovich, A.A. New data on the distribution of genus Ammophila... Acta Biologica Sibirica, 2019, 5(1), 44-46

Ammophila apicalis Brullé, 1839; Ammophila terminata F. Smith, 1856; Ammophila mocsaryi Frivaldszky, 1876; Ammophila minuta Frivaldszkyi, 1877; Ammophila rhaetica Kohl, 1879; Ammophila julii Fabre, 1879; Ammophila kirgisica F. Morawitz, 1891; Ammophila gegen Tsuneki, 1971.

MATERIAL: $3 \circ$ Point №4, 1 + Point №5.

DISTRIBUTION: Russia: EP (C, E, S, CR), UR, WS (OM, NS, AL), ES (TU, KR, IR, BR, ZB). - Europe (WE, NE, SE, EE), N Africa, TR, Central Asia, KZ, MN, China (NC, NW).

\section{Conclusion}

As a result of our study check-list of circumpolar regions Western Siberia species was complied. That apparently makes up less than $50 \%$ of actual Sphecidae fauna of the Okrug.

\section{Acknowledgments}

The author thanks Professor Pierre Rasmont and Baptiste Martinet for helping in collecting the material.

\section{References}

Antropov, A.V., Astafurova, Yu.V., Belokobylskij, S.A., Byvaltsev, A.M., Danilov, Yu.N., Dubovikoff, D.A., Fadeev, K.I., Fateryga, A.V., Kurzenko, N.V., Lelej, A.S., Levchenko, T.V., Loktionov, V.M., Mokrousov, M.V., Nemkov P.G., Proschalykin M.Yu., Rosa, P., Sidorov, D.A., Sunducov, Yu.N., Yusupov, Z.M., \& Zaytsev, L.A. (2017). Annotated catalogue of Hymenoptera of Russia. Volume 1. Symphyta and Apocrita: Aculeata. Proceedings of the Zoological institute RAS, 6. (Suppl.).

Danilov, Yu.N. (2014). Review of Sphecidae wasps (Hymenoptera: Apoidea) of Siberia. Part 2. Keys to genera and species. Euroasian entomological journal, 13(6), 511-521. (in Russian)

Pulawski, W.J. (2018) Catalog of Sphecidae. California Academy of Sciences, San Francisco. Retrieved October 11, 2018, from http://researcharchive.calacademy.org/research/entomology/entomology_resources/hymeno-pterra/sphecidae/genera/-

Ammophila.pdf

\section{Citation:}

Baghirov, R.T-o., \& Nesterovich, A.A. (2019). New data on the distribution of digger wasps of the genus Ammophila W. Kirby, 1798 (Hymenoptera, Sphecidae). Acta Biologica Sibirica, 5 (1), 44-46.

Submitted: 10.12.2018. Accepted: 19.01.2019

cross ref http://dx.doi.org/10.14258/abs.v5.i1.5189

(C) 2019 by the authors. Submitted for possible open access publication under the terms and conditions of the Creative Commons Attribution (CC BY) license (http://creativecommons.org/licenses/by/4.0/). 